\title{
MODIFYING EFFECTS OF LACTOFERRIN IN VITRO ON MOLECULAR PHENOTYPE OF HUMAN BREAST CANCER CELLS
}

\author{
V.F. Chekhun ${ }^{1}$, I.V. Zalutskii ${ }^{2}$ L.A. Naleskina ${ }^{1}$, N.Yu. Lukianova ${ }^{1, *}$, \\ T.M. Yalovenko', T.V. Borikun ${ }^{1}$, S.O. Sobchenko ${ }^{1}$, I.V. Semak ${ }^{3}$, V.S. Lukashevich ${ }^{2}$ \\ ${ }^{1}$ R.E. Kavetsky Institute of Experimental Pathology, Oncology and Radiobiology, NAS of Ukraine, Kyiv \\ 03022, Ukraine \\ ${ }^{2}$ State Scientific Institution “Institute of Physiology”, NAS of Belarus, \\ Minsk 220072, Republic of Belarus \\ ${ }^{3}$ Institution of Education “Belarusian State University”, Minsk 220030, Republic of Belarus
}

\begin{abstract}
Aim: To assess the role of endogenous lactoferrin (LF) in the formation of the molecular phenotype of human breast cancer (BC) cell lines with varying degrees of malignancy, including cisplatin/doxorubicin resistant cell lines, and identify possible impact of exogenous LF. Materials and Methods: 5 breast cell lines of different origin - MCF-10 A, MCF-7, including doxorubicin/ cisplatin resistant ones, T47D, MDA-MB-231, and MDA-MB-468. Immunocytochemistry: expression of LF, Ki-67, adhesion molecules E- and N-cadherin, CD44, CD24 rating the invasive potential of cells. Results: Expression of LF in human BC cell lines varies. It is associated with the heterogeneity of molecular profiles of cell lines in terms of adhesion. A link has been established between the level of LF expression in the resistant cell line MCF-7/CP and MCF-7/Dox, features of their molecular profile and invasive properties. Exogenous LF was shown to be capable of modifying the molecular profile and invasive properties of all the studied cell lines including resistant ones (MCF-7/CP and MCF-7/Dox). Conclusions: The sensitivity of cytostatic-resistant cell lines (MCF-7/CP and MCF-7/Dox) tends to increase under the influence of exogenous LF. It is likely that this effect is due to LF-mediated inhibition of the expression of proteins associated with drug resistance.
\end{abstract}

Key Words: lactoferrin, cell lines of human breast cancer, molecular phenotype, proliferative activity, invasive potential, adhesion molecules.

\section{INTRODUCTION}

Undoubtedly, breast cancer (BC) will soon discontinue being a uniform nosological form as it used to be until recently. This is due to the fact that a vast majority of experts in fundamental and clinical oncology have recently recognized that $\mathrm{BC}$ is a set of diseases with different "natural evolution" and clinical features and that they should no longer be treated in a "stereotypical manner" (in particular, this applies to using chemotherapeutic agents); instead, tactics of individualized treatment of patients should be applied [1]. Such a change in the views on BC a malignancy which belongs to the most common ones among the women population - is due to the fact that numerous clinical observations have proved the presence of distinctive associative links between certain morphological and functional characteristics, molecular-genetic profiles of tumors and clinical manifestations of the tumor process in BC patients [2, 3].

It has been clearly shown that the existence of intertumor and intracellular heterogeneity of populations of malignantly transformed cells leads to a diversity of clinical pictures of $\mathrm{BC}$ and oftentimes to a failure to achieve a response after a standard treatment in individuals with similar characteristics, such as age, prevalence of the process, form or degree of histological differentiation [4].

Submitted: April 22, 2015.

${ }^{\star}$ Correspondence: E-mail: lu_na_u@rambler.ru

Abbreviations used: $\mathrm{BC}$ - breast cancer; LF - lactoferrin; MDR multidrug resistance; $\mathrm{NChT}$ - neoadjuvant chemotherapy.
A biological phenomenon of intracellular heterogeneity, which is caused by genetic instability, is multi-dimensional and is considered to be a key factor in determining the vector of the cancer process, both at the onset and during the realization of various forms of tumor progression, i.e. the aggressiveness of the disease $[5,6]$. Many researchers believe that intracellular heterogeneity plays a crucial role in the rate of neoplasm development, its oncogenic potential, and cell survival in the conditions of dynamic microenvironment [7-9]. The heterogeneity within the same tumor manifests itself in the form of differences between various groups of cells in terms of morphological structure, genetic and epigenetic status, as well as variability in the expression of various markers, including molecular genetic ones [10-12].

Intracellular heterogeneity makes the tumor cell population diverse in terms of biological potential of malignancy and helps it to expand by way of dissemination, colonization, metastasizing, and also enhances its resistance to antitumor effects, primarily to chemotherapy. There are reports showing that numerous subpopulations of cells are present in a tumor from the very onset; they feature varying functional characteristics and differ in the degree of sensitivity or resistance to particular medicines [13, 14]. Also, it was established that intratumoral morphological heterogeneity of $\mathrm{BC}$ is associated with the expression of multidrug resistance (MDR) genes and the efficiency of neoadjuvant chemotherapy (NChT). In particular, it was shown that tumors featuring alveolar structures 
are characterized by a high level and up-regulation of main MDR genes and, at the same time, by a poor response to the NChT [15].

It has been proven that the parenchymatous component of $\mathrm{BC}$ with the different biological aggressiveness contains cells with varying phenotypic and molecular genetic characteristics. Based on this, methods have been developed for selective therapeutic interventions, which should be based on the results of experimental studies [16, 17]. The basis for this are numerous reports on existing relation between the intratumoral morphological heterogeneity and the molecular subtypes in terms of expression of ER, $\mathrm{PR}$ and HER2/neu as the most intensively studied area of clinical molecular research in $B C[18,19]$. In particular, it was noted that the "triple negative BC" more often features one single type of morphological structures, while luminal BC mainly features 5 types of structures: tubular, solid, trabecular, alveolar and discrete groups of tumor cells [20]. The comparison of the achievements of fundamental science with the specific characteristics of the disease course is an important link between the proposed hypotheses about the mechanisms that may underlie the disease development and the objective assessment of their likelihood in practical cancer care.

Over the last decade, along with enhanced research aimed to confirm the achievements of molecular genetics concerning the existence of $\mathrm{BC}$ heterogeneity in terms of molecular phenotype, as well as their introduction into clinical practice, considerable efforts have been made to increase our knowledge about the role of metal-containing proteins in malignant transformation and tumor progression, as important components of metabolism, which are actively involved in various processes of cells' vital activity [21]. Our interest is focused on fundamental research of one of the most poorly studied among proteins of such origin - lactoferrin (LF) $[22,23]$ - in terms of expression patterns in BC cells varying in phenotypic profile and malignancy degree, and the possibilities to modify the malignant potential of these cells when incubated with recombinant human LF. This interest is due to the fact that our own clinical observations of two geographical location populations of $\mathrm{BC}$ patients for the first time showed that the LF expression level is characterized by inter-tumor heterogeneity, as it was detected only in 48.0 and $53.6 \%$ of the cases, respectively. Furthermore, it was proved that the survival rate among women with LF-positive tumors was higher than in tumors of patients without LF expression [24].

Based on the above, our aim was to conduct experiments in vitro to establish LF expression patterns in cell lines of human $\mathrm{BC}$ with varying malignancy potential, including those resistant to cisplatin and doxorubicin, and to check if the phenotype of these cells can be modified by culturing them with exogenous LF.

\section{MATERIALS AND METHODS}

Cell lines and drug treatment. The studies were conducted in an in vitro system on 5 cell lines of human mammary of different origins: MCF-10A - immortalized cells of normal human mammary; MCF-7 - invasive breast ductal carcinoma; T47D - metastatic breast ductal carcinoma; MDA-MB-231 and MDAMB-468 - metastatic breast adenocarcinoma.

T47D cells were cultured in RPMI- 1640 medium (Sigma) supplemented by bovine insulin $(0.2 \mathrm{U} / \mathrm{ml})$ and $10 \%$ fetal bovine serum (FBS). MCF- 7 cells were grown in medium Dulbeccos Modified Eagles Medium (Sigma) supplemented by recombinant human insulin $(0.01 \mathrm{mg} / \mathrm{ml})$ and $10 \%$ FBS. MDA-MB-231 and MDAMB-468 cells were cultured in Leibovitz's L- 15 medium (Sigma) supplemented by $10 \%$ FBS. MCF-10A cells were grown in MEBM medium (Lonza) supplemented by cholera toxin $(100 \mathrm{ng} / \mathrm{ml})$. All cultures were grown on glass cover slips in humidified atmosphere with $5 \% \mathrm{CO}_{2}$ at $37^{\circ} \mathrm{C}$. The cells were provided by the Bank of Human and Animal Tissue Lines of the R.E. Kavetsky Institute of Experimental Pathology, Oncology and Radiobiology of the National Academy of Sciences of Ukraine.

The resistant variants MCF-7/Doxand MCF-7/CP were originated by growing initial MCF-7 cells with raising concentrations of cisplatin (from 0,01 to $6 \mu \mathrm{g} / \mathrm{ml}$ ) or doxorubicin (from 0.1 to $32 \mu \mathrm{g} / \mathrm{ml}$ ), respectively. Cisplatin and doxorubicin were added twice a week after reseeding. Every 2 months, cell survival was analyzed by MTT assay. IC I0 $_{50}$ values for MCF- 7 and MCF-7/CP cells were 0.25 and $1 \mu \mathrm{g} / \mathrm{ml}$ of cisplatin, respectively, and for MCF- 7 and MCF-7/Dox cells -0.5 and $8 \mu \mathrm{g} / \mathrm{ml}$ of doxorubicin, respectively. Therefore, MCF-7/CP were 4 times as much resistant to the cytotoxic effect of cisplatin and MCF-7/DOX cells were 16 times as much resistant to the cytotoxic effect doxorubicin as compared with the initial MCF-7 cells.

MTT assay. Sensitivity to antitumor drugs (cisplatin and doxorubicin) was measured every two months using standard MTT-colorimetric test with 3-[4,5, dimethylthiasol-2-1]-2,5-diphenyltetrasolium bromide ("Sigma", Germany) [25].

We used a wide range of immunocytochemical methods that allow establishing the molecular profile of malignantly transformed cells: assessment of the expression level of adhesion molecules such as E-cadherin, $\mathrm{N}$-cadherin, $\mathrm{CD} 44, \mathrm{CD} 24$, proliferative activity by expression of $\mathrm{Ki}-67$. The LF expression in tumor cells and their invasive properties were studied. Cells for immunocytochemical studies were grown on glass cover slips, fixed in cooled mixture of methanol:acetone (1:1) at $--20{ }^{\circ} \mathrm{C}$ for $120 \mathrm{~min}$, washed in PBS and incubated with $1 \%$ BSA for 20 min. Primary monoclonal antibodies: anti-lactoferrin (ThermoScientific, USA); antiKi-67 (DakoCytomation, Denmark); anti-CD24 (NeoMarkers, USA); anti-CD44 (Diagnostic BioSystems, USA); anti-E-cadherin (ThermoScientific, USA), anti-CD325 (N-Cadherin) (DakoCytomation, Denmark) were diluted in the blocking buffer and kept at a room 
temperature for one hour, followed by incubation with UltraVision LP Detection System (Lab Vision, Thermo Scientific) for 10 and $15 \mathrm{~min}$; after the washing, the immune reaction was visualized by using DAB Quanto (Thermo Scientific). When immunocytochemical reactions were completed, the cells were stained with hematoxylin by Mayer for 10-15 s and placed in Faramount Aqueous Mounting Medium (DakoCytomation, Denmark). Evaluation of the results was carried out in 3 visual fields by light microscopy (x1000, oil immersion) using the classical H-Score method:

$$
S=1 \times N_{1}^{+}+2 \times N_{2}{ }^{+}+3 \times N_{3}{ }^{+},
$$

where $\mathrm{S}$ is " $\mathrm{H}$-Score" index, $\mathrm{N}_{1}{ }^{+}, \mathrm{N}_{2}{ }^{+}$and $\mathrm{N}_{3}{ }^{+}$are numbers of cells with low, medium or high levels of marker expression.

It should be noted that in previous studies we have identified biomolecular markers that characterize the metastatic potential and invasive activity of BC cells of abovementioned lines [18].

Invasive activity. The invasive activity was investigated using a standard method, according to manufacturer's recommendations (BD Biosciences). For this purpose, the matrigel membranes (BD BioCoat ${ }^{\mathrm{TM}}$ Matrige $^{\mathrm{TM}}$ Invasion Chamber, Bedford, MA) with pore sizes of 8 microns were used. In the upper part of the membrane, the cell suspension was placed in a concentration of $5 \cdot 10^{4} \mathrm{cells} /$ well in culture medium without the addition of bovine serum. In the lower part, the standard culture medium supplemented with $10 \%$ FBS was introduced. The cells were then incubated under standard conditions at temperature of $37{ }^{\circ} \mathrm{C}$ in humidified atmosphere with $5 \% \mathrm{CO}_{2}$ during 48 hours. The membranes were then fixed with methanol, followed by staining with crystal violet $B$. The number of cells on outside of membrane was evaluated using light microscopy ( $\times 100$, oil immersion).

LF preparation (obtaining). Milk of goats transfected with human LF gene (joint development of SPC on Livestock Farming of the National Academy of Sciences of Belarus and the Institute of Gene Biology, Russian Academy of Sciences) was subjected to the standard procedure of casein removing. Chromatography was performed using a medium pressure liquid chromatograph ACTAFPLCsystem (Amersham-Pharmacia). Milk serum was applied to a HiPrep 16/10 SP HL column (Amersham-Pharmacia) that was prewashed with equilibration buffer ( $20 \mathrm{mM}$ phosphate $\mathrm{Na} ; 0.4 \mathrm{M} \mathrm{NaCl}$;
$\mathrm{pH}$ 6.5) at a rate $1 \mathrm{ml} / \mathrm{min}$. LF was eluted with a linear gradient $0.4 \mathrm{M}-1 \mathrm{M} \mathrm{NaCl}$. Fractions containing LF were concentrated and desalted using ultrafiltration cassettes Vivaflow 50 (Sartorius) with a molecular weight cut-off of $30 \mathrm{kDa}$. The resulting concentrate was lyophilized on Lyophilize ALPHA 1-4 LDplus. The purity of obtained LF (>99\%) was assessed by denaturing gel electrophoresis in polyacrylamide gel in the presence of sodium dodecyl sulfate.

Recombinant human LF, which was used in experiments in vitro, was diluted in PBS and added directly to the culture medium at a final concentration of $100 \mu \mathrm{g} / \mathrm{mL}$. Cells were cultured with LF during $72 \mathrm{~h}$.

Statistical analysis was performed with STATISTICA 6.0 software (StatSoft Inc., USA). The all data were obtained in triplicate experiments. The values were expressed as means \pm standard deviation (SD). Student's $t$-test was used to evaluate the significance of the differences between groups. A value of $p<0.05$ was accepted as statistically significant.

\section{RESULTS AND DISCUSSION}

Immunocytochemical determination of LF revealed that this metal-containing protein shows varying expression levels in the tested BC cell lines (Table 1). The highest LF expression was observed in cell lines T47D and MCF-7 that possess low invasive activity, which according to the molecular phenotype is caused by the increased adhesion properties of these cells due to the high expression level of E-cadherin and low expression level of CD44, in the absence of CD24 expression. Levels of $\mathrm{N}$-cadherin expression were definitely moderate.

The lowest level of LF expression was seen in cell line MDA-MB-468, that is characterized by the highest rates of invasion activity, which according to their molecular profile is explained by the higher expression of adhesion molecules CD44 and CD24 as compared with those in cell lines T47D and MCF-7 with a low invasive potential. At the same time, expression of E-cadherin has been at the values range typical for cells with a low invasion activity. Levels of the LF expression were slightly higher in cell line MDA-MB-231, which by its invasive properties were inferior to cell line MDAMB-468, and among the tested cell lines had the lowest level of E-cadherin expression, the highest expression of CD44 molecules and the positive response to CD24.

Table 1. Changes in the molecular profile of $B C$ cells with different molecular phenotype and invasive properties caused by cultivation with LF

\begin{tabular}{|c|c|c|c|c|c|c|c|}
\hline Cell lines & Level of LF expression & Cells' proliferative activity & CD24 & CD44 & E-cadherin & $\mathrm{N}$-cadherin & Invasion, $\times 10^{3}$ cells \\
\hline T47D & $285.0 \pm 2.1$ & $89.0 \pm 1.4$ & 0 & $79.0 \pm 3.1$ & $251.0 \pm 5.0$ & $53.0 \pm 2.4$ & $0.081 \pm 0.002$ \\
\hline $\mathrm{T} 47 \mathrm{D}+\mathrm{LF}$ & - & $63.0 \pm 0.8^{*}$ & 0 & $43.0 \pm 1.1^{*}$ & $263.0 \pm 3.2^{*}$ & $49.0 \pm 2.0$ & $0.061 \pm 0.005^{\star}$ \\
\hline MCF-7 & $251.0 \pm 2.3$ & $291.0 \pm 2.4$ & 0 & $72.0 \pm 2.8$ & $268.0 \pm 4.6$ & $59.0 \pm 1.6$ & $0.087 \pm 0.005$ \\
\hline MCF-7 + LF & - & $170.0 \pm 2.1^{*}$ & 0 & $32.0 \pm 2.3^{*}$ & $283.0 \pm 3.6^{\star}$ & $56.0 \pm 1.9$ & $0.070 \pm 0.004$ \\
\hline MDA-MB-231 & $92.0 \pm 1.9$ & $268.0 \pm 1.9$ & $34.0 \pm 2.6$ & $298.0 \pm 0.7$ & $49.0 \pm 1.3$ & $31.0 \pm 0.7$ & $0.192 \pm 0.021$ \\
\hline MDA-MB-231 + LF & - & $130.0 \pm 2.3^{*}$ & $34.0 \pm 2.6$ & $164.0 \pm 1.7^{*}$ & $148.0 \pm 2.9^{*}$ & $48.0 \pm 0.9^{*}$ & $0.153 \pm 0.014^{*}$ \\
\hline MDA-MB4-68 & $70.0 \pm 1.6$ & $249.0 \pm 2.7$ & $153.0 \pm 4.0$ & $167.0 \pm 5.0$ & $259.0 \pm 2.5$ & $18.0 \pm 0.8$ & $0.340 \pm 0.011$ \\
\hline MDA-MB-468 + LF & - & $119.0 \pm 2.8^{\star}$ & $110.0 \pm 2.1^{*}$ & $96.0 \pm 3.0^{*}$ & $293.0 \pm 2.8^{*}$ & $31.0 \pm 0.9^{*}$ & $0.190 \pm 0.021^{*}$ \\
\hline MCF-10A & $157.0 \pm 1.8$ & $191.0 \pm 2.3$ & $63.0 \pm 1.8$ & $174.0 \pm 4.3$ & $149.0 \pm 1.1$ & $31.0 \pm 1.6$ & $0.240 \pm 0.016$ \\
\hline$M C F-10 A+L F$ & - & $130.0 \pm 2.7^{*}$ & $42.0 \pm 1.6^{\star}$ & $110.0 \pm 2.3^{*}$ & $189.0 \pm 1.9^{\star}$ & $28.0 \pm 1.1^{\star}$ & $0.176 \pm 0.009$ \\
\hline MCF-7/CP & $105.0 \pm 1.7$ & $143.0 \pm 2.5$ & 0 & $176.0 \pm 2.1$ & $57.0 \pm 1.9$ & $179.0 \pm 1.7$ & $0.280 \pm 0.010$ \\
\hline $\mathrm{MCF}-7 / \mathrm{CP}+\mathrm{LF}$ & 0 & $87.0 \pm 1.5^{\star}$ & 0 & $98.0 \pm 2.2^{\star}$ & $115.0 \pm 1.6^{\star}$ & $143.0 \pm 1.8^{\star \star}$ & $0.154 \pm 0.013^{*}$ \\
\hline MCF-7/Dox & $67.0 \pm 1.9$ & $95.0 \pm 1.8$ & 0 & $234.0 \pm 2.3$ & $28.0 \pm 2.3$ & $238.0 \pm 1.9$ & $0.352 \pm 0.022$ \\
\hline MCF-7/Dox + LF & 0 & $62.0 \pm 1.6^{\star}$ & 0 & $110.0 \pm 2.3^{*}$ & $73.0 \pm 1.9^{\star}$ & $169.0 \pm 1.8^{*}$ & $0.142 \pm 0.008^{\star}$ \\
\hline
\end{tabular}

Note: ${ }^{*} p<0.05$ compared with cell lines without LF. 
In immortalized cells of human mammary cell line MCF-10A without signs of malignant transformation, we found a high LF expression. By its invasive properties the cells of that line occupied intermediate position between cell lines MDA-MB-468 and MDA-MB-231. In these cells, there was a high level of CD44 and CD24 expression and, at the same time, a fairly high expression of E-cadherin (see Table 1). It should be noted that the proliferative activity was equally high in the tested cell lines and did not significantly affect both the levels of the LF expression and invasive activity. Thus, according to our findings, the level of LF expression in human BC cell lines of different origins is associated with their invasive properties. At the same time, the invasive activity of the tested cell lines is caused by the expression of various adhesion molecules: the weak manifestations of invasion associates with the high level of E-cadherin expression and the lack of CD24 expression, while cells with an active invasion showed a shift towards increased expression levels of CD44 and CD24. The above mentioned differences are the direct evidence of interlinear heterogeneity of BC cells in both the level of LF expression and the molecular profile which determines their invasive properties.

Along with this, we evaluated the features of LF expression in cells of the two resistant $B C$ lines, namely cisplatin-resistant (MCF-7/CP), and doxorubicinresistant (MCF-7/Dox) (see Table 1). According to our findings, the LF expression level in cell line MCF-7/ CP was much higher than that in cell line MCF-7/ Dox. At the same time, invasive activity was more pronounced in cell line MCF-7/Dox, which was characterized by a low expression of this metal-containing protein. In both lines, we found low levels of E-cadherin expression, which were significantly lower in cell line MCF-7/Dox; high expression levels of CD44 and $\mathrm{N}$-cadherin, significantly higher in cells resistant to doxorubicin; and absence of CD24 expression in both cell lines. The proliferative activity was lower in cell line MCF-7/Dox.

In the literature, there are data obtained in vivo and in vitro systems, which show that exogenous LF can affect the rate of tumor cells growth. Thus, in vivo experiments showed that systemic effects of LF resulted in the inhibited growth of squamous cell skin carcinoma transplanted to normal mice; while when the tumor was transplanted into immune-deficient mice such effect was absent. The same tendency was seen when metastatic colon cancer was transplanted to normal mice and athymic animals [25]. In animals with subcutaneously transplanted highly metastatic colon carcinoma, the number of lung metastases decreased by $48.0 \%$ after LF administration. In the same model, it was shown that the reduction of the metastasis intensity was accompanied by the increased Interleukin 18 production and the subsequent induction of interferon, to which the authors attribute the antimetastatic effect of LF. In addition, the researchers concluded that the activation of Interleukin 18 was associated with the activation of caspase 1 [26]. Using small angle $X$-ray scattering, light scattering and ablation, it was convincingly shown that DNA, nucleotides and oligosaccharides cause the formation of certain LF oligomers, which differ in their antitumor activities and influence on the immune system [27]. Finally, in studies on clinical material received from patients with intestinal polyposis it has been found that a longterm LF administration in high doses was not toxic and resulted in slower growth of polyps. The studies have shown that after the action of digestive enzymes the LF cleaves forming lactoferritin which retains the biological activity of LF. In experiments conducted on cell cultures aiming to determine the mechanisms of LF action, it was shown that in some types of tumor cells LF is capable of stimulating apoptosis and inhibiting cell proliferation by blocking the transition from G1 phase to S phase of the cell cycle [28, 29]. At the same time, in one of the reports it was noticed that in human BC cell line MCF-7 LF did not inhibit the tumor cell growth if it was cultured with this ironcontaining protein [30].

A scarce and controversial data on this issue have prompted us to analyze whether LF really has a modifying effect on the molecular profile and invasive properties of tested BC cell lines by culturing them with exogenous LF (see Table 1). As a result of this phase of research, it was found that culturing all tested human $\mathrm{BC}$ cell lines with LF resulted in the decrease of their malignant potential, but the manifestations of the action of the iron-containing protein were ambiguous. In particular, there was a decline in proliferative activity levels and a significant weakening of the cells' invasive properties due to enhanced intercellular adhesion (significant increase in the E-cadherin expression and decrease in the CD44 expression). The CD24 expression was slightly reduced, and the expression of $\mathrm{N}$-cadherin did not change substantially. It should be noted that the most pronounced effects of changes in the molecular profile and invasive properties under the LF influence were observed in the BC cell lines MDA-MB-231 and MDA-MB-468, which had the highest malignant potential according to the investigated parameters that was identified before incubating the cells with this iron-containing protein.

Analysis of the biological effect of LF on the molecular profile of cytostatic-resistant BC cells that characterizes their adhesive properties and invasive activity, allowed us identifying a number of common features and essential peculiarities in each of the tested lines. Thus, a common feature for both resistant lines exposed to LF was a significant inhibition of proliferation activity, decrease of the E-cadherin expression level, significant decline of CD44 expression, as well as reduction of $\mathrm{N}$-cadherin expression. Directly in cell line MCF-7/CP, the level of E-cadherin expression increased twice. The CD44 expression decreased by 1.7 times, as well as the index of their invasive activity. In cell line MCF-7/Dox these indices changed even more dramatically: the expression of E-cadherin 
increased twice, the expression of CD44 and invasive activity decreased by 2.5 times.

The obtained data became the basis for the study of the LF impacts on the most important component of molecular phenotype of resistant cells - expression of proteins associated with MDR: namely P-gp and GST. Immunohistochemical studies showed that in cell line MCF-7/CP, which was not subjected to the LF influence, P-gp expression was not detected, and the GST expression level was $276.0 \pm 2.2$ (Table 2). In cell line MCF-7/Dox, which also served as the similar control, on the contrary, the P-gp expression was determined (285.0 \pm 1.9 ), while there was no expression of GST. Culturing the cells of both lines with LF caused a substantial reduction of expression of the tested proteins: GST halved in cell line MCF-7/CP and $\mathrm{P}$-gp halved in cell line MCF-7/Dox.

Table 2. The changes in expression of proteins associated with drug resistance in $\mathrm{BC}$ cells with the phenotype of drug resistance caused by cultivating with LF

\begin{tabular}{ccccc}
\hline Resistance & \multicolumn{4}{c}{ Cell line } \\
\cline { 2 - 5 } marker & MCF-7/CP & MCF-7/CP + LF & MCF-7/Dox & MCF-7/Dox + LF \\
\hline P-gp & 0 & 0 & $285.0 \pm 1.9$ & $120.0 \pm 1.6^{*}$ \\
GST & $276.0 \pm 2.2$ & $135.0 \pm 2.4^{\star}$ & 0 & 0 \\
\hline
\end{tabular}

Note: ${ }^{\star} \mathrm{p}<0.03$ compared with cell line without LF.

The next step in the identification of the LF influence on cytostatic-resistant MCF-7 cell line was the study of the ability of this iron-containing protein to increase cells' sensitivity to anticancer drugs. Our findings showed that the doxorubicin $\mathrm{IC}_{50}$ for cell line MCF-7/Dox after incubation with LF increased twice as compared with the cells of that line not exposed to $L F$, indicating a substantial increase in their sensitivity to this cytostatic (Table 3 ). A similar phenomenon of increasing the cells' sensitivity caused by their culturing with LF was found in cell line MCF-7/CP towards cisplatin too. Less pronounced manifestations of cells sensitivity enhancement to cytostatics in terms of $\mathrm{IC}_{50}$ were noticed in cell line MCF-7/Dox towards cisplatin and in MCF-7/CP towards doxorubicin. Thus, the contact of LF with cell lines MCF-7, which is resistant, among others, to doxorubicin and cisplatin, is able of significantly improving their sensitivity to the above-mentioned cytostatics.

Table 3. Increased sensitivity of $B C$ cells to anticancer drugs caused by cultivation with LF

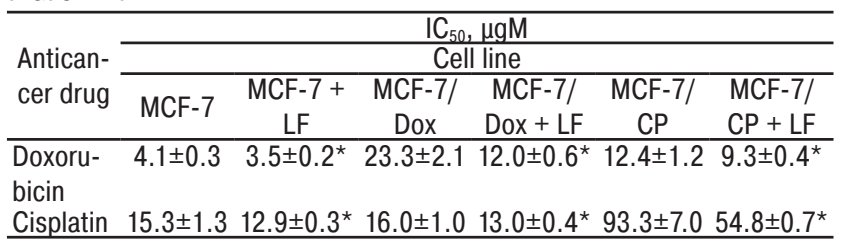

Note: ${ }^{*} \mathrm{p}<0.05$ compared with cell line without LF.

Our findings do not contradict the results of other studies that evidence that the manifestation of LF effects on some properties of tumor cells in vitro can be regarded as anti-carcinogenic effect [31]. The above gives hope for the future prospects of the prescription of LF in the complex personalized therapy of BC patients. At the same time, our own experimental studies indicate that, at the stage of establishing $\mathrm{BC}$ diagnosis, it is needed to conduct, along with the LF expression evaluation, also the assessment of certain molecular markers' expression, which can help identify the malignant potential of the neoplasm, and on this basis to focus on the individualized prescription of LF.

The fact that LF in vitro has the ability of increasing tumor cells' sensitivity to cytostatics, especially resistant ones, warrants further studies using exogenous $\mathrm{LF}$ in vivo to confirm the effect of overcoming MDR.

A significant obstacle to large-scale research and multi-center clinical trials is the absence of recombinant human LF in the international market. At present, the developments of genetically engineered constructs with gene of human LF are being carried out actively for production of this protein on a commercial scale $[32,33]$. The successful resolution of this issue will help approach the time when the use of LF in the complex treatment of $\mathrm{BC}$ patients will become a reality.

\section{CONCLUSIONS}

1. It was shown that the tested human $B C$ cell lines are heterogeneous in terms of LF expression.

2. The various manifestations of LF expression in the tested human $\mathrm{BC}$ cell lines are associated with the heterogeneity of molecular profile of these cell lines in terms of adhesion.

3. The highest level of the LF expression was observed in cell lines T47D and MCF-7, which have the lowest manifestations of invasive properties. Cell lines MDA-MB-231 and MDA-MB-468 with highly invasive activities feature low levels of the LF expression.

4. The relationship between the LF expression levels in resistant cell lines MCF-7/CP and MCF-7/Dox and the features of their molecular profile and invasive properties was established.

5. The modifying effect of LF on the molecular profile and invasive properties of all tested lines, including resistant ones - MCF-7/CP and MCF-7/Dox, was established.

6. The inhibitory effect of LF on the expression of proteins associated with MDR was established in $B C$ cells with MDR phenotypes.

7. As a result of the cultivation with LF of initial human $\mathrm{BC}$ cell lines and BC cell lines resistant to cytostatics, their sensitivity to doxorubicin and cisplatin increased, which was most pronounced in the resistant cell lines: MCF-7/CP and MCF-7/Dox. This effect is due to the inhibited expression of MDR associated proteins.

\section{ACKNOWLEDGMENTS}

The study was supported by joint scientific project of the NAS of Ukraine and the NAS of Belarus 2015-2016 "Molecular-Biological Effects and Mechanisms of Lactoferrin Action on Tumor Cells in vitro and in vivo".

\section{REFERENCES}

1. Polyak K, Dana-Farber. Heterogeneity in breast cancer. J Clin Invest 2011; 121: 3786-8.

2. Albain U, Paik S, Van't Veer L. Prediction of adjuvant chemotherapy benefit in endocrine responsive early breast cancer using multigene assays. The Breast 2009; 18: 141-5. 
3. Curigliano G, Viale G, Bagnardi V, et al. Clinical relevance of HER2 overexpression/amplification in patients with small tumor size and node negative breast cancer. J Clin Oncol 2009; 27: 5693-9.

4. Semiglazov VF, Semiglazov VV. Breast cancer screening. Prakticheskaya Onkologiya 2010; 11: 60-5 (in Russian).

5. Chekhun VF, Sherban SD, Savtsova ZD. Tumor heterogeneity - a dynamic state. Oncology 2012; 14: 4-12 (in Russian).

6. Yi-Hsuan Hsiao, Ming-Chih Chou, Carol Fowler, et al. Breast cancer heterogeneity: mechanisms, proofs, and implications. J Cancer 2010; 1: 6-13.

7. Michor F, Polyak K. The origins and implications of intratumor heterogeneity. Cancer Prev Res 2010; 3: 1361-4.

8. Andrechek ER, Nevins JR. Mouse models of cancers: opportunities to address heterogeneity of human cancer and evaluate therapeutic strategies. J Mol Med 2010; 88: $1095-100$.

9. Somasundaram R, Villanueva J, Herlyn M. Intratumoral heterogeneity as a therapy resistance mechanism: role of melanoma subpopulations. Adv Pharmacol 2012; 65: 335-59.

10. Kulagina ESh. Epidemiological and molecular aspects of breast cancer. Prakt Onkol 2010; 11: 203-16 (in Russian).

11. Nemtsova MV, Paltseva EM, Babayan AYu, et al. Molecular genetic analysis of clonal intratumoral heterogeneity in colorectal carcinomas. Mol Biol 2008; 42: 1040-7 (in Russian).

12. Baldus SE, Schaefer KL, Engers R, et al. Prevalence and heterogeneity of KRAS, BRAF, and PIK3CA mutations in primary colorectal adenocarcinomas and their corresponding metastases. Clin Cancer Res 2010; 16: 790-9.

13. Saunders NA, Simpson F, Thompson EW, et al. Role of intratumoural heterogeneity in cancer drug resistance: molecular and clinical perspectives. EMBO Mol Med 2012; 4: 675-84.

14. Geraschenko TS, Denisov EV, Litvyakov NV, et al. Intratumoral heterogeneity: nature and biological significance. Biohimiya 2013; 78: 1531-49 (in Russian).

15. Tsyganov MM, Geraschenko TS, Denisov EV, et al. Intratumoral morphological heterogeneity of breast cancer: expression of multidrug resistance genes and effectiveness of neoadjuvant antitumor chemotherapy. Sib Onkol J 2013; (1 (Suppl)): 93-4 (in Russian).

16. Geraschenko TS, Zavyalova MV, Denisov EV, et al. Intratumoral morphological heterogeneity of invasive ductal breast cancer: development and molecular-genetic features. Medit Akad J 2012; 12: 66-8 (in Russian).

17. Perelmuter VM, Zavyalova MV, Vtorushin SV, et al. Relations between morphological heterogeneity of infiltrative ductal breast cancer with different forms of tumor progression. Sib Onkol J 2007; 23: 58-63 (in Russian).

18. Onitilo AA, Engel JM, Greenlee RT, et al. Breast cancer subtypes based on ER/PR and Her2 expression: com- parison of clinicopathologic features and survival. Clin Med Res 2009; 7: 4-13.

19. Guo L, Meng J, Yilamu D, et al. Significance of ER $\beta$ expression in different molecular subtypes of breast cancer. Diagn Pathol 2014; 9: 1-6.

20. Zavyalova MV, Denisov EV, Tashireva LA, et al. Phenotypic drift as a cause for intratumoral morphological heterogeneity of invasive ductal breast carcinoma not otherwise specified. Biores Open Access 2013; 2: 148-54.

21. Chekhun VF, Shpilevaya SI. Role of endogenous iron in development of tumor sensitivity to antitumor therapy. Voprosy Onkologii 2010; 56: 251-61 (in Russian).

22. Berlov MN. Lactoferrin from dog neutrophils: extraction, physico-chemical and antimicrobic properties. Biohimia 2007; 72: 551-9 (in Russian).

23. Kang J-F, Li X-L, Zhou RY, et al. Bioinformatics analysis of lactoferrin gene for several species. Biochem Genetics 2008; 46: 312-22.

24. Lagutin AY, Sobchenko SA, Zadvorny TV. Evaluation of lactofferin levels as a marker of breast cancer biological activity. Shevchenkivska Vesna 2014; 7: 42 (in Ukrainian).

25. Hollander D, Ni J. Application of the MTT-assay to functional studies of mouse intestinal intraepithelial lymphocytes. J Clin Lab Anal 1996; 10: 42-52.

26. Tsuda H, Sekine K, Takasuka N. Prevention of colon carcinogenesis and carcinoma metastasis by orally administered bovin lactoferrin in animals. Biofactors 2000; 12: 83-8.

27. Kuhara I, Ligo M, Itoh T. Orally administered lactoferrin exerts an antimetastatic effect and enhances production of IL-18 in the intestinal epithelium. Nutr Cancer 2000; 38: $192-9$.

28. Nevinskii AG, Soboleva SE, Tuzikov FV. DNA, oligosaccharides, and mononucleotides stimulate oligomerization of human lactoferrin. Mol Recogn 2009; 22: 330-42.

29. Xiao Y, Monitto CL, Minhas KM, et al. Lactoferrin down-regulates $\mathrm{G} 1$ cyclin-dependent kinases during growth arrest of head and neck cancer cells. Clin Cancer Res 2004; 10: 8683-6.

30. Yang N, Stensen W, Svendsen JS, et al. Enhanced antitumor activity and selectivity of lactoferrin-derived peptides. J Pept Res 2002; 60: 187-97.

31. Wolf JS, Li D, Taylor RJ, O’Malley BW Jr. Lactoferrin inhibits growth of malignant tumors of the head and neck. ORL J Otorhinolaryngol Relat 2003; 65: 245-9.

32. Chekhun V, Bezdenezhnykh N, Shvets J, et al. Expression of biomarkers related to cell adhesion, metastasis and invasion of breast cancer cell lines of different molecular subtype. Exp Oncol 2013; 35: 174-9.

33. Naroditskiy BS, Shmarov MM, Logunov DYu, et al. Method of synthesis of recombinant human lactoferrin. Russian Patent 2008; N 2340674 (in Russian). 